\title{
SYSTEMS OF DIVISION PROBLEMS FOR DISTRIBUTIONS
}

\author{
BY \\ B. ROTH
}

\begin{abstract}
Suppose $\left(f_{i j}\right)_{1 \leqq i, j \leqq p}$ is a $p \times p$ matrix of real-valued infinitely (respectively $m$-times continuously) differentiable functions on an open subset $\Omega$ of $R^{n}$. Then $\left(f_{i j}\right)_{1 \leqq i, j \leqq p}$ maps the space of $p$-tuples of distributions on $\Omega$ (respectively distributions of order $\leqq m$ on $\Omega$ ) into itself. In the present paper, the $p \times p$ matrices $\left(f_{i j}\right)_{1 \leqq i, j \leqq p}$ for which this mapping is onto are characterized in terms of the zeros of the determinant of $\left(f_{i j}\right)_{1 \leqq i, j \leqq p}$ when the $f_{i}$ are infinitely differentiable on $\Omega \subset R^{1}$ and when the $f_{i j}$ are $m$-times continuously differentiable on $\Omega \subset R^{n}$. Finally, partial results are obtained when the $f_{t}$ are infinitely differentiable on $\Omega \subset R^{n}$ and extensions are made to $p \times q$ systems of division problems for distributions.
\end{abstract}

1. Introduction. Let $\mathscr{E}^{m}(\Omega)$ denote the algebra of real-valued $m$-times continuously differentiable functions on an open set $\Omega$ in $R^{n}$ equipped with the topology of uniform convergence of all derivatives of order $\leqq m$ on all compact subsets of $\Omega$. Here $0 \leqq m \leqq \infty$ and $\mathscr{E}^{\infty}(\Omega)$ will often be denoted by $\mathscr{E}(\Omega) . \mathscr{E}^{m}(\Omega)$ is a Fréchet space, that is, a complete metrisable locally convex topological vector space. Let $\mathscr{D}_{K}^{m}(\Omega)$ denote the subspace of $\mathscr{E}^{m}(\Omega)$ consisting of all functions with support in the compact set $K \subset \Omega$ equipped with the relative topology and let $\mathscr{D}^{m}(\Omega)$ denote the inductive limit of the $\mathscr{D}_{K}^{m}(\Omega), K$ a compact subset of $\Omega$. Here $0 \leqq m \leqq \infty$ and $\mathscr{D}^{\infty}(\Omega)$ will often be denoted by $\mathscr{D}(\Omega)$. For $m<\infty, \mathscr{D}^{\prime m}(\Omega)$ (respectively $\mathscr{E}^{\prime m}(\Omega)$ ), the dual space of $\mathscr{D}^{m}(\Omega)$ (respectively $\mathscr{E}^{m}(\Omega)$ ), is the space of distributions of order $\leqq m$ on $\Omega$ (respectively the space of distributions with compact support of order $\leqq m$ on $\Omega$ ). And $\mathscr{D}^{\prime}(\Omega)$ (respectively $\mathscr{E}^{\prime}(\Omega)$ ), the dual space of $\mathscr{D}(\Omega)$ (respectively $\mathscr{E}(\Omega)$ ), is the space of distributions on $\Omega$ (respectively the space of distributions with compact support on $\Omega$ ).

For $F=\left(f_{i j}\right)_{1 \leqq i \leqq p, 1 \leqq j \leqq q}$ where $f_{i j} \in \mathscr{E}^{m}(\Omega)$, define $F:\left[\mathscr{D}^{\prime m}(\Omega)\right]^{q} \rightarrow\left[\mathscr{D}^{\prime m}(\Omega)\right]^{p}$ by

$$
F\left(S_{1}, \ldots, S_{q}\right)=\left(\sum_{j=1}^{q} f_{1 j} S_{j}, \ldots, \sum_{j=1}^{q} f_{p j} S_{j}\right) .
$$

Let $F_{c}:\left[\mathscr{E}^{\prime m}(\Omega)\right]^{q} \rightarrow\left[\mathscr{E}^{\prime m}(\Omega)\right]^{p}$ be the restriction of $F$ to $\left[\mathscr{E}^{\prime m}(\Omega)\right]^{q}$. Let $F^{\prime}:\left[\mathscr{D}^{m}(\Omega)\right]^{p} \rightarrow\left[\mathscr{D}^{m}(\Omega)\right]^{q}$ (respectively $F_{c}^{\prime}:\left[\mathscr{E}^{m}(\Omega)\right]^{p} \rightarrow\left[\mathscr{E}^{m}(\Omega)\right]^{q}$ ) be the transpose of $F$ (respectively $F_{c}$ ). Then $F^{\prime}=F_{c}^{\prime}=\left(f_{i j}^{\prime}\right)_{1 \leqq i \leqq q, 1 \leqq j \leqq p}$ where $f_{i j}^{\prime}=f_{j i}, 1 \leqq i \leqq q$, $1 \leqq j \leqq p$.

Presented to the Society, March 27, 1970; received by the editors April 20, 1970 and, in revised form, July $13,1970$.

AMS 1969 subject classifications. Primary 4640; Secondary 4630.

Key words and phrases. Spaces of distributions, division of distributions, zeros of finite order, the Lojasiewicz inequality. 
When does $\operatorname{im}(F)=\left[\mathscr{D}^{\prime m}(\Omega)\right]^{p}$, that is, when does there exist a solution $\left(S_{1}, \ldots, S_{q}\right) \in\left[\mathscr{D}^{\prime m}(\Omega)\right]^{q}$ to the $p \times q$ system of division problems

$$
\begin{aligned}
& f_{11} S_{1}+\cdots+f_{1 q} S_{q}=T_{1} \\
& \vdots \quad \vdots \quad \vdots \quad \vdots \\
& f_{p 1} S_{1}+\cdots+f_{p q} S_{q}=T_{p}
\end{aligned}
$$

for every $\left(T_{1}, \ldots, T_{p}\right) \in\left[\mathscr{D}^{\prime m}(\Omega)\right]^{p}$ ?

In $\S 2$ we show that for $p=q, m<\infty$, and $\Omega \subset R^{n}$, im $(F)=\left[\mathscr{D}^{\prime m}(\Omega)\right]^{p}$ if and only if $\operatorname{det}(F)$ never vanishes. In $\S 3$ it is shown that for $p=q$ and $\Omega \subset R^{1}$, the following are equivalent:

(a) $\operatorname{im}(F)=\left[\mathscr{D}^{\prime}(\Omega)\right]^{p}$.

(b) $\operatorname{det}(F)$ has zeros of finite order.

(c) $\operatorname{det}(F)$ is not identically zero in any component of $\Omega$ and satisfies the Lojasiewicz inequality.

In $\S 4$ we prove that for $p=q$ and $\Omega \subset R^{n}, n>1$, (a) implies (b), (a) implies (c), and (b) does not imply (a). The referee has pointed out that (c) does not imply (a) for $n>1$. And in $\S 5$ we dispense with our restriction that $p=q$ and obtain for the general system of division problems results analogous to those of $\S 2, \S 3$, and $\S 4$.

2. Systems of division problems in $\mathscr{D}^{\prime m}(\Omega), m<\infty, \Omega \subset R^{n}$. For $F=\left(f_{i j}\right)_{1 \leqq i, j \leqq p}$ where $f_{i j} \in \mathscr{E}^{m}(\Omega), m<\infty, \Omega \subset R^{n}$, we here show that im $(F)=\left[\mathscr{D}^{\prime m}(\Omega)\right]^{p}$ if and only if $\operatorname{det}(F)$ never vanishes. The proof reduces the $p \times p$ system of division problems to a $1 \times p$ system. We begin with a lemma involving $1 \times p$ systems in which Malgrange's extension [4] to submodules of a theorem of Whitney [8] describing the closed ideals in $\mathscr{E}^{m}(\Omega)$ is used.

LeMma 2.1. Suppose $f_{1}, \ldots, f_{p} \in \mathscr{E}^{m}(\Omega), m<\infty, \Omega \subset R^{n}$, and define

$$
F_{c}:\left[\mathscr{E}^{\prime m}(\Omega)\right]^{p} \rightarrow \mathscr{E}^{\prime m}(\Omega)
$$

by $F_{c}\left(S_{1}, \ldots, S_{p}\right)=f_{1} S_{1}+\cdots+f_{p} S_{p} . \quad$ If $f_{i}(a)=0,1 \leqq i \leqq p$, where $a \in \Omega$, then $\partial_{1}^{m} \delta_{a} \notin \operatorname{im}\left(F_{c}\right)$.

Proof. Suppose $f_{i}(a)=0,1 \leqq i \leqq p$, where $a=\left(a_{1}, \ldots, a_{n}\right) \in \Omega$. We assume that $F_{c}\left(S_{1}, \ldots, S_{p}\right)=\partial_{1}^{m} \delta_{a}$ where $S_{1}, \ldots, S_{p} \in \mathscr{E}^{\prime m}(\Omega)$ and obtain a contradiction.

Define $f$ by $f\left(x_{1}, \ldots, x_{n}\right)=\left(x_{1}-a_{1}\right)^{l}+\cdots+\left(x_{n}-a_{n}\right)^{l}$ where $l$ is an even integer greater than $m$. Then

$$
F_{c}\left(f S_{1}, \ldots, f S_{p}\right)=f\left(\partial_{1}^{m} \delta_{a}\right)=0 .
$$

Thus $\left(f S_{1}, \ldots, f S_{p}\right) \in \operatorname{ker}\left(F_{c}\right)=M^{\perp}$ where $M=\operatorname{im}\left(F_{c}^{\prime}\right)=\left\{\left(f_{1} g, \ldots, f_{p} g\right): g \in \mathscr{E}^{m}(\Omega)\right\}$ is a submodule of the $\mathscr{E}^{m}(\Omega)$-module $\left[\mathscr{E}^{m}(\Omega)\right]^{p}$. Hence $\left(S_{1}, \ldots, S_{p}\right) \in(f M)^{\perp}$ where $f M$ is the submodule $\left\{\left(f g_{1}, \ldots, f g_{p}\right):\left(g_{1}, \ldots, g_{p}\right) \in M\right\}$.

For $x \in \Omega$, let $J_{x}^{m}$ denote the ideal in $\mathscr{E}^{m}(\Omega)$ consisting of all function which vanish at $x$ together with all derivatives of order $\leqq m$ and let $T_{x}^{m}$ denote the natural mapping of $\left[\mathscr{E}^{m}(\Omega)\right]^{p}$ onto $\left[\mathscr{E}^{m}(\Omega)\right]^{p} /\left[J_{x}^{m}\right]^{p}$. 
Define $g$ by $g\left(x_{1}, \ldots, x_{n}\right)=\left(x_{1}-a_{1}\right)^{m}$. Then $T_{a}^{m}(f M)$ and $T_{a}^{m}(g M)$ are both the zero submodule of $\left[\mathscr{E}^{m}(\Omega)\right]^{p} /\left[J_{a}^{m}\right]^{p}$. And for $x \in \Omega, x \neq a$, we have $T_{x}^{m}(g M) \subset T_{x}^{m}(f M)$ since $f(x) \neq 0$. By Whitney's theorem for submodules [4, Corollary 1.6, p. 25], we conclude that $\mathrm{cl}(g M) \subset \mathrm{cl}(f M)$, and hence that $(f M)^{\perp} \subset(g M)^{\perp}$.

Therefore $\left(S_{1}, \ldots, S_{p}\right) \in(g M)^{\perp}$ and thus $\left(g S_{1}, \ldots, g S_{p}\right) \in M^{\perp}=\operatorname{ker}\left(F_{c}\right)$. Hence

$$
0=F_{c}\left(g S_{1}, \ldots, g S_{p}\right)=g\left(\partial_{1}^{m} \delta_{a}\right)
$$

But $g\left(\partial_{1}^{m} \delta_{a}\right) \neq 0$ since $g\left(x_{1}, \ldots, x_{n}\right)=\left(x_{1}-a_{1}\right)^{m}$. This contradiction completes the proof.

THEOREM 2.1. Suppose $F=\left(f_{i j}\right)_{1 \leqq i, j \leqq p}$ where $f_{i j} \in \mathscr{E}^{m}(\Omega), m<\infty, \Omega \subset R^{n}$. Then the following are equivalent:

(a) $\operatorname{im}(F)=\left[\mathscr{D}^{\prime m}(\Omega)\right]^{p}$.

(b) $\operatorname{im}\left(F_{c}\right)=\left[\mathscr{E}^{\prime m}(\Omega)\right]^{p}$.

(c) $\operatorname{det}(F(x)) \neq 0$ for all $x \in \Omega$.

(d) $\operatorname{det}(F) \mathscr{D}^{\prime m}(\Omega)=\mathscr{D}^{\prime m}(\Omega)$.

Proof. (a)implies(b). If $F\left(S_{1}, \ldots, S_{p}\right)=\left(T_{1}, \ldots, T_{p}\right)$ where $\left(S_{1}, \ldots, S_{p}\right) \in\left[\mathscr{D}^{\prime m}(\Omega)\right]^{p}$ and $\left(T_{1}, \ldots, T_{p}\right) \in\left[\mathscr{E}^{\prime m}(\Omega)\right]^{p}$, then for an appropriate $\psi \in \mathscr{E}^{m}(\Omega)$ with compact support we have $F\left(\psi S_{1}, \ldots, \psi S_{p}\right)=\left(T_{1}, \ldots, T_{p}\right)$ where

$$
\left(\psi S_{1}, \ldots, \psi S_{p}\right) \in\left[\mathscr{E}^{\prime m}(\Omega)\right]^{p} .
$$

(b) implies (c). Suppose $\operatorname{im}\left(F_{c}\right)=\left[\mathscr{E}^{\prime m}(\Omega)\right]^{p}$. We suppose that $\operatorname{det}(F(a))=0$ for some $a \in \Omega$ and obtain a contradiction. Let $r$ be the rank of the matrix $F(a)$. If $r=0$, then $f_{i j}(a)=0,1 \leqq i, j \leqq p$. By hypothesis, there exist $S_{1}, \ldots, S_{p} \in \mathscr{E}^{\prime m}(\Omega)$ with $F_{c}\left(S_{1}, \ldots, S_{p}\right)=\left(\partial_{1}^{m} \delta_{a}, 0, \ldots, 0\right)$, contradicting Lernma 2.1 .

Suppose $1 \leqq r<p$. For simplicity assume that $\operatorname{det}\left(f_{i j}\right)_{1 \leqq i, j \leqq r}$ is nonzero at $a$. Choose $T \in \mathscr{E}^{\prime m}(\Omega)$ such that $(-1)^{r} \operatorname{det}\left(f_{i j}\right)_{1 \leqq i, j \leqq r} T=\partial_{1}^{m} \delta_{a}$. By hypothesis, there exist $S_{1}, \ldots, S_{p} \in \mathscr{E}^{\prime m}(\Omega)$ with

$$
\begin{gathered}
f_{11} S_{1}+\cdots+f_{1 p} S_{p}=0 \\
\vdots \quad \vdots \quad \vdots \quad \\
f_{r 1} S_{1}+\cdots+f_{r p} S_{p}= \\
f_{(r+1) 1} S_{1}+\cdots+f_{(r+1) p} S_{p}=T .
\end{gathered}
$$

For $1 \leqq i \leqq r+1$, multiply the $i$ th row of this system of equations by $(-1)^{i+1}$ times the determinant of the matrix $\left(f_{i j}\right)_{1 \leqq i \leqq r+1,1 \leqq j \leqq r}$ with the $i$ th row deleted. Adding the $r+1$ equations thus obtained, we find that the coefficient $g_{j}$ of $S_{j}$ is

$$
g_{j}=\operatorname{det}\left(\begin{array}{cccc}
f_{1 j} & f_{11} & \cdots & f_{1 r} \\
\vdots & \vdots & & \vdots \\
f_{(r+1) j} & f_{(r+1) 1} & \cdots & f_{(r+1) r}
\end{array}\right) \text {. }
$$


For $1 \leqq j \leqq r, g_{j}$ is identically zero since two columns are the same. For $r<j \leqq p, g_{j}(a)=0$ since the rank of $F(a)$ is $r$. Thus

$$
g_{1} S_{1}+\cdots+g_{p} S_{p}=(-1)^{r} \operatorname{det}\left(f_{i j}\right)_{1 \leqq i, j \leqq r} T=\partial_{1}^{m} \delta_{a}
$$

where $g_{j}(a)=0,1 \leqq j \leqq p$, which contradicts Lemma 2.1 .

(c) implies (d). Division by a nonzero function is always possible.

(d) implies (a). Since it is always the case that $\left[\operatorname{det}(F) \mathscr{D}^{\prime m}(\Omega)\right]^{p} \subset \operatorname{im}(F)$, $\operatorname{det}(F) \mathscr{D}^{\prime m}(\Omega)=\mathscr{D}^{\prime m}(\Omega)$ implies im $(F)=\left[\mathscr{D}^{\prime m}(\Omega)\right]^{p}$.

3. Systems of division problems in $\mathscr{D}^{\prime}(\Omega), \Omega \subset R^{1}$. For $F=\left(f_{i j}\right)_{1 \leqq i, j \leqq p}$ where $f_{i j} \in \mathscr{E}(\Omega), \Omega \subset R^{1}$, we here show the equivalence of $\operatorname{im}(F)=\left[\mathscr{D}^{\prime}(\Omega)\right]^{p}$, the zeros of $\operatorname{det}(F)$ are of finite order, and det $(F)$ satisfies the Lojasiewicz inequality but is not identically zero in any component of $\Omega$. The proof uses the closed range theorem for Fréchet spaces due to Dieudonné and Schwartz [1], the open mapping theorem for Fréchet spaces, and some results of the author [6] concerning the relationship between zeros of finite order and the Lojasiewicz inequality.

Consider $\mathscr{E}(\Omega), \Omega \subset R^{n}$. For each compact set $K \subset \Omega$ and integer $l \geqq 0$, let

$$
|f|_{K, l}=\sup \left\{\left|\partial_{1}^{\alpha_{1}} \cdots \partial_{n}^{\alpha_{n}} f(x)\right|: x \in K, \alpha_{1}+\cdots+\alpha_{n} \leqq l\right\}
$$

for $f \in \mathscr{E}(\Omega)$. The seminorms $|\cdot|_{K, l}$ define the topology of $\mathscr{E}(\Omega)$. For

$$
f=\left(f_{1}, \ldots, f_{p}\right) \in[\mathscr{E}(\Omega)]^{p},
$$

let

$$
|f|_{K, l}=\max \left\{\left|f_{1}\right|_{K, l}, \ldots,\left|f_{p}\right|_{K, l}\right\} .
$$

The seminorms $|\cdot|_{K, l}$ define the topology of $[\mathscr{E}(\Omega)]^{p}$.

Malgrange [4, p. 88] first recognized the usefulness of the open mapping theorem in establishing the Lojasiewicz inequality. To establish the Lojasiewicz inequality in this way, the following fact is needed. For any compact set $K \subset \Omega$, integer $l \geqq 0$, and nonempty closed subset $Z$ of $\Omega$, there exists a constant $C>0$ such that if $x \in K-Z$, then there exists $h_{x} \in \mathscr{E}(\Omega)$ with $h_{x}(x)=1, h_{x}=0$ in a neighborhood of $Z$, and

$$
\left|h_{x}\right|_{K, l} \leqq C /[d(x, Z)]^{l} .
$$

Here $d(x, Z)=\inf \{d(x, z): z \in Z\}$ where $d$ is the Euclidean metric. Merely let $\psi$ be an infinitely differentiable function with support in the unit ball and $\psi(0)=1$. Then $h_{x}(y)=\psi((y-x) / \delta)$ has the desired properties when $\delta=d(x, Z) / 2$.

Suppose $f \in \mathscr{E}(\Omega), \Omega \subset R^{1}$. A zero of $f$ is of finite order if some derivative of $f$ does not vanish at the zero.

Finally, we need a lemma which will te used again in $\S 4$ and $\S 5$.

Lemma 3.1. Suppose $F=\left(f_{i j}\right)_{1 \leqq i, j \leqq p}$ where $f_{i j} \in \mathscr{E}^{m}(\Omega), 0 \leqq m \leqq \infty, \Omega \subset R^{n}$. Then $F_{c}^{\prime}:\left[\mathscr{E}^{m}(\Omega)\right]^{p} \rightarrow\left[\mathscr{E}^{m}(\Omega)\right]^{p}$ is one-to-one if and only if $\operatorname{det}(F)$ is not identically zero in any open subset of $\Omega$. 
Proof. Suppose that $\operatorname{det}(F)=\operatorname{det}\left(F_{c}^{\prime}\right)=0$ in some open subset $U$ of $\Omega$ where $F_{c}^{\prime}=\left(f_{i j}^{\prime}\right)_{1 \leqq i, j \leqq p}, f_{i j}^{\prime}=f_{j i}$. Let $r$ be the maximum rank of $F_{c}^{\prime}(x)$ for $x \in U$. If $r=0$, then $f_{i j}^{\prime}=0$ in $U$ for $1 \leqq i, j \leqq p$ and clearly $F_{c}^{\prime}$ is not one-to-one.

Suppose $1 \leqq r<p$. For simplicity assume that $\operatorname{det}\left(f_{i j}^{\prime}\right)_{1 \leqq i, j \leqq r}$ is nonzero at $a \in U$. Therefore $\operatorname{det}\left(f_{i j}^{\prime}\right)_{1 \leqq i, j \leqq r}$ is nonzero in an open neighborhood $N$ of $a$ with $N \subset U$. Then

$$
\begin{aligned}
& f_{11}^{\prime} g_{1}+\cdots+f_{1 r}^{\prime} g_{r}=f_{1(r+1)}^{\prime} \\
& \begin{array}{ccc}
\vdots & \vdots & \vdots \\
f_{r 1}^{\prime} g_{1}+\cdots+f_{r r}^{\prime} g_{r} & = & f_{r(r+1)}^{\prime}
\end{array}
\end{aligned}
$$

can be solved in $N$ by Cramer's rule for functions $g_{1}, \ldots, g_{r} \in \mathscr{E}^{m}(N)$.

Choose $\psi \in \mathscr{E}^{m}(\Omega)$ with $\psi \not \equiv 0$ and $\operatorname{supp}(\psi) \subset N$. Let $h_{1}=\psi g_{1}, \ldots, h_{r}=\psi g_{r}, h_{r+1}$ $=-\psi$, and $h_{j}=0, r+1<j \leqq p$. Then $\left(h_{1}, \ldots, h_{p}\right) \in \operatorname{ker}\left(F_{c}^{\prime}\right)$. For

$$
F_{c}^{\prime}\left(h_{1}, \ldots, h_{p}\right)=\left(\sum_{j=1}^{p} f_{1 j}^{\prime} h_{j}, \ldots, \sum_{j=1}^{p} f_{p j}^{\prime} h_{j}\right)
$$

And for $1 \leqq i \leqq p$

$$
\sum_{j=1}^{p} f_{i j}^{\prime} h_{j}=f_{i 1}^{\prime} \psi g_{1}+\cdots+f_{i r}^{\prime} \psi g_{r}-f_{i(r+1)}^{\prime} \psi
$$

Since supp $(\psi) \subset N$, we have $\sum_{j=1}^{p} f_{i j}^{\prime} h_{j}=0$ in $\Omega-N$ for $1 \leqq i \leqq p$. And in $N$, we have

$$
\sum_{j=1}^{p} f_{i j}^{\prime} h_{j}=(-1)^{r+1} \psi \operatorname{det}\left(\begin{array}{ccc}
f_{i 1}^{\prime} & \cdots & f_{i(r+1)}^{\prime} \\
f_{11}^{\prime} & \cdots & f_{1(r+1)}^{\prime} \\
\vdots & & \vdots \\
f_{r 1}^{\prime} & \cdots & f_{r(r+1)}^{\prime}
\end{array}\right) / \operatorname{det}\left(f_{i j}^{\prime}\right)_{1 \leqq i, j \leqq r}
$$

For $1 \leqq i \leqq r$, the determinant of the matrix in the numerator equals zero since two rows are the same, and for $r<i \leqq p$, it equals zero since the maximum rank of $F_{c}^{\prime}$ in $N$ is $r$. Since $\left(h_{1}, \ldots, h_{p}\right) \in \operatorname{ker}\left(F_{c}^{\prime}\right)$ and $h_{r+1}=-\psi \not \equiv 0$, we conclude that $F_{c}^{\prime}$ is not one-to-one.

Conversely, suppose that $\operatorname{det}(F)=\operatorname{det}\left(F_{c}^{\prime}\right)$ is not identically zero in any open subset of $\Omega$. Suppose $\left(g_{1}, \ldots, g_{p}\right) \in \operatorname{ker}\left(F_{c}^{\prime}\right)$. Then

$$
\begin{aligned}
& f_{11}^{\prime} g_{1}+\cdots+f_{1 p}^{\prime} g_{p}=0 \\
& \vdots \quad \vdots \quad \vdots \quad \vdots \\
& f_{p 1}^{\prime} g_{1}+\cdots+f_{p p}^{\prime} g_{p}=0 \text {. }
\end{aligned}
$$

Consider $j, 1 \leqq j \leqq p$. For $1 \leqq i \leqq p$, multiply the $i$ th row of this system of equations by the cofactor of $f_{i j}^{\prime}$ in $F_{c}^{\prime}$. Adding the equations thus obtained, we find that $\operatorname{det}\left(F_{c}^{\prime}\right) g_{j}=0$. Hence $g_{j}=0$ since $\operatorname{det}\left(F_{c}^{\prime}\right)$ is not identically zero in any open subset of $\Omega$. Therefore $F_{c}^{\prime}$ is one-to-one. 
THEOREM 3.1. Suppose $F=\left(f_{i j}\right)_{1 \leqq i, j \leqq p}$ where $f_{i j} \in \mathscr{E}(\Omega), \Omega \subset R^{1}$. Then the following are equivalent:

(a) $\operatorname{im}(F)=\left[\mathscr{D}^{\prime}(\Omega)\right]^{p}$.

(b) $\operatorname{im}\left(F_{c}\right)=\left[\mathscr{E}^{\prime}(\Omega)\right]^{p}$.

(c) $F_{c}^{\prime}$ is one-to-one and im $\left(F_{c}^{\prime}\right)$ is closed in $[\mathscr{E}(\Omega)]^{p}$.

(c) $\operatorname{det}(F)$ is not identically zero in any component of $\Omega$ and $\operatorname{det}(F)$ satisfies the Lojasiewicz inequality, that is, for each compact set $K \subset \Omega$ there exists a constant $C>0$ and an integer $l \geqq 0$ such that

$$
|\operatorname{det}(F(x))| \geqq C[d(x, Z)]^{l} \text { for all } x \in K
$$

where $Z=\{x \in \Omega$ : $\operatorname{det}(F(x))=0\}$. (Here $d(x, Z)=1$ for all $x$ if $Z=\varnothing$.)

(e) The zeros of $\operatorname{det}(F)$ are of finite order.

(f) $\operatorname{det}(F) \mathscr{D}^{\prime}(\Omega)=\mathscr{D}^{\prime}(\Omega)$.

Proof. (a) implies (b). If $F\left(S_{1}, \ldots, S_{p}\right)=\left(T_{1}, \ldots, T_{p}\right)$ where $\left(S_{1}, \ldots, S_{p}\right) \in\left[\mathscr{D}^{\prime}(\Omega)\right]^{p}$ and $\left(T_{1}, \ldots, T_{p}\right) \in\left[\mathscr{E}^{\prime}(\Omega)\right]^{p}$, then for an appropriate $\psi \in \mathscr{E}(\Omega)$ with compact support, we have $F\left(\psi S_{1}, \ldots, \psi S_{p}\right)=\left(T_{1}, \ldots, T_{p}\right)$ where $\left(\psi S_{1}, \ldots, \psi S_{p}\right) \in\left[\mathscr{E}^{\prime}(\Omega)\right]^{p}$.

(b) implies (c). By the closed range theorem for Fréchet spaces [1, Theorem 7 , p. 92], if $\operatorname{im}\left(F_{c}\right)=\left[\mathscr{E}^{\prime}(\Omega)\right]^{p}$, then $F_{c}^{\prime}:[\mathscr{E}(\Omega)]^{p} \rightarrow[\mathscr{E}(\Omega)]^{p}$ is one-to-one and has closed range.

(c) implies (d). If $F_{c}^{\prime}$ is one-to-one, then Lemma $3.1 \mathrm{implies}$ that $\operatorname{det}(F)$ is not identically zero in any component of $\Omega$. We now assume that im $\left(F_{c}^{\prime}\right)$ is closed in $[\mathscr{E}(\Omega)]^{p}$ and prove that $\operatorname{det}(F)=\operatorname{det}\left(F_{c}^{\prime}\right)$, where $F_{c}^{\prime}=\left(f_{i j}^{\prime}\right)_{1 \leqq i, j \leqq p}, f_{i j}^{\prime}=f_{j i}$, satisfies the Lojasiewicz inequality. The case $Z=\varnothing$ being trivial, we assume $Z$ is nonempty.

Let $K \subset \Omega$ be a compact set. $F_{c}^{\prime}$ is a continuous linear mapping of the Fréchet space $[\mathscr{E}(\Omega)]^{p}$ onto the Fréchet space im $\left(F_{c}^{\prime}\right)$ and therefore $F_{c}^{\prime}$ is an open mapping. Hence there exist a constant $C>0$, an integer $l \geqq 0$, and a compact set $K^{\prime} \subset \Omega$ with $K \subset K^{\prime}$ such that if $h \in \operatorname{im}\left(F_{c}^{\prime}\right)$, then there exists $g \in[\mathscr{E}(\Omega)]^{p}$ with $F_{c}^{\prime}(g)=h$ and $|g|_{K, 0} \leqq C|h|_{K^{\prime}, l}$.

For the compact set $K^{\prime} \subset \Omega$, the integer $l \geqq 0$, and the nonempty closed subset $Z$ of $\Omega$ there exists a constant $C^{\prime}>0$ such that if $x \in K^{\prime}-Z$, then there exists $h_{x} \in \mathscr{E}(\Omega)$ with $h_{x}(x)=1, h_{x}=0$ in a neighborhood of $Z$, and $\left|h_{x}\right|_{K^{\prime}, l} \leqq C^{\prime} /[d(x, Z)]^{l}$.

By induction, we now prove the property $P(k), 1 \leqq k \leqq p$, where $P(k)$ states that there is a constant $C_{k}>0$ such that the maximum of the absolute values of the determinants of the $k \times k$ submatrices of $F_{c}^{\prime}(x)$ is greater than or equal to $C_{k}[d(x, Z)]^{k l}$ for all $x \in K$. Observe $P(p)$ states that there is a constant $C_{p}>0$ such that

$$
|\operatorname{det}(F(x))|=\left|\operatorname{det}\left(F_{c}^{\prime}(x)\right)\right| \geqq C_{p}[d(x, Z)]^{p l} \text { for all } x \in K \text {. }
$$

First, we prove $P(1)$. Consider $x \in K-Z$ and let $h=\left(h_{x}, 0, \ldots, 0\right)$. Then $h \in \operatorname{im}\left(F_{c}^{\prime}\right)$ since $h_{x}=0$ in a neighborhood of $Z$. Therefore there exists

with $F_{c}^{\prime}(g)=h$ and

$$
g=\left(g_{1}, \ldots, g_{p}\right) \in[\mathscr{E}(\Omega)]^{p}
$$

$$
|g|_{K, 0} \leqq C|h|_{K^{\prime}, l} \leqq C C^{\prime} /[d(x, Z)]^{l}
$$


Since $F_{c}^{\prime}(g)=h$, we have $f_{11}^{\prime}(x) g_{1}(x)+\cdots+f_{1 p}^{\prime}(x) g_{p}(x)=h_{x}(x)=1$ so for some $j, 1 \leqq j \leqq p$, we have $\left|f_{1 j}^{\prime}(x) g_{j}(x)\right| \geqq 1 / p$. Thus

$$
1 /\left|f_{1 \jmath}^{\prime}(x)\right| \leqq p\left|g_{j}(x)\right| \leqq p|g|_{K, 0} \leqq p C C^{\prime} /[d(x, Z)]^{l} .
$$

Therefore for each $x \in K-Z$, there exists $j, 1 \leqq j \leqq p$, such that

$$
\left|f_{1 j}^{\prime}(x)\right| \geqq[d(x, Z)]^{l} / p C C^{\prime} .
$$

This proves $P(1)$.

Now assume that $P(k)$ holds where $1 \leqq k<p$ and prove $P(k+1)$. Consider $x \in K-Z$. For simplicity assume that

$$
\left|\operatorname{det}\left(f_{i j}^{\prime}(x)\right)_{1 \leqq i, j \leqq k}\right| \geqq C_{k}[d(x, Z)]^{k l} .
$$

Let $h=\left(h_{1}, \ldots, h_{p}\right)$ where $h_{k+1}=h_{x}$ and $h_{i}=0, i \neq k+1$. Then $h \in \operatorname{im}\left(F_{c}^{\prime}\right)$ since $h_{x}=0$ in a neighborhood of $Z$. Therefore there exists $g=\left(g_{1}, \ldots, g_{p}\right) \in[\mathscr{E}(\Omega)]^{p}$ with $F_{c}^{\prime}(g)=h$ and $|g|_{K, 0} \leqq C|h|_{K^{\prime}, l} \leqq C C^{\prime} /[d(x, Z)]^{l}$. Since $F_{c}^{\prime}(g)=h$, we have

$$
\begin{aligned}
& f_{11}^{\prime}(x) g_{1}(x)+\cdots+f_{1 p}^{\prime}(x) g_{p}(x)=0 \\
& f_{k 1}^{\prime}(x) g_{1}(x)+\cdots+f_{k p}^{\prime}(x) g_{p}(x)=0 \\
& f_{(k+1) 1}^{\prime}(x) g_{1}(x)+\cdots+f_{(k+1) p}^{\prime}(x) g_{p}(x)=h_{x}(x)=1 \text {. }
\end{aligned}
$$

For $1 \leqq i \leqq k+1$, multiply the $i$ th row of this system of equations by $(-1)^{i+1}$ times the determinant of the matrix $\left(f_{i j}^{\prime}(x)\right)_{1 \leqq i \leqq k+1,1 \leqq j \leqq k}$ with the $i$ th row deleted. Adding the equations thus obtained, we find that the coefficient of $g_{j}(x)$ for $1 \leqq j \leqq p$ is

$$
d_{j}=\operatorname{det}\left(\begin{array}{cccc}
f_{1 j}^{\prime}(x) & f_{11}^{\prime}(x) & \cdots & f_{1 k}^{\prime}(x) \\
\vdots & \vdots & & \vdots \\
f_{(k+1) j}^{\prime}(x) & f_{(k+1) 1}^{\prime}(x) & \cdots & f_{(k+1) k}^{\prime}(x)
\end{array}\right)
$$

Since $d_{j}=0$ for $1 \leqq j \leqq k$, we have

$$
\sum_{j=k+1}^{p} d_{j} g_{j}(x)=(-1)^{k} \operatorname{det}\left(f_{i j}^{\prime}(x)\right)_{1 \leqq i, j \leqq k} .
$$

Therefore for some $j, k+1 \leqq j \leqq p$, we have

$$
\left|d_{j} g_{j}(x)\right| \geqq\left|\operatorname{det}\left(f_{i j}^{\prime}(x)\right)_{1 \leqq i, j \leqq k}\right| / p \geqq C_{k}[d(x, Z)]^{k l} / p .
$$

Since $\left|g_{j}(x)\right| \leqq|g|_{K, 0}$, we have

$$
1 /\left|d_{j}\right| \leqq p|g|_{K, 0} / C_{k}[d(x, Z)]^{k l} \leqq p C C^{\prime} / C_{k}[d(x, Z)]^{(k+1) l} .
$$

And since $\left|d_{j}\right|$ for $k+1 \leqq j \leqq p$ is the absolute value of the determinant of a $(k+1) \times(k+1)$ submatrix of $F_{c}^{\prime}(x)$, we have proved $P(k+1)$. 
Since $P(p)$ states that $\operatorname{det}\left(F_{c}^{\prime}\right)=\operatorname{det}(F)$ satisfies the Lojasiewicz inequality in $K$, we conclude that if im $\left(F_{c}^{\prime}\right)$ is closed in $[\mathscr{E}(\Omega)]^{p}$, then det $(F)$ satisfies the Lojasiewicz inequality.

The equivalence of (d), (e), and (f) is a result of the author [6, Corollary 6.2] for connected $\Omega$ and the extension to arbitrary open subsets of $R^{n}$ is immediate.

(f) implies (a). Since it is always the case that $\left[\operatorname{det}(F) \mathscr{D}^{\prime}(\Omega)\right]^{p} \subset \operatorname{im}(F)$, $\operatorname{det}(F) \mathscr{D}^{\prime}(\Omega)=\mathscr{D}^{\prime}(\Omega)$ implies im $(F)=\left[\mathscr{D}^{\prime}(\Omega)\right]^{p}$.

4. Systems of division problems in $\mathscr{D}^{\prime}(\Omega), \Omega \subset R^{n}$. It is known that for $f \in \mathscr{E}(\Omega)$, $\Omega \subset R^{n}, f_{D^{\prime}}(\Omega)=\mathscr{D}^{\prime}(\Omega)$ if $f$ is a polynomial (Hörmander [2, Theorem 4, p. 568]) and, more generally, if $f$ is real analytic in $\Omega$ and not identically zero in any component of $\Omega$ (Lojasiewicz [3, p. 130]). Furthermore, the results of Malgrange [5, Theorem 1, p. 23-01] together with Lemma 3.1 imply that for $F=\left(f_{i j}\right)_{1 \leqq i, j \leqq p}$ where the $f_{i j}$ are real analytic in $\Omega$, if $\operatorname{det}(F)$ is not identically zero in any component of $\Omega$, then $\operatorname{im}(F)=\left[\mathscr{D}^{\prime}(\Omega)\right]^{p}$. However, a necessary and sufficient condition that $\operatorname{im}(F)$ $=\left[\mathscr{D}^{\prime}(\Omega)\right]^{p}$, where $F=\left(f_{i j}\right)_{1 \leqq i, j \leqq p}, f_{i j} \in \mathscr{E}(\Omega), \Omega \subset R^{n}, n>1$, seems to be unknown, even for $p=1$.

Several necessary conditions that $\operatorname{im}(F)=\left[\mathscr{D}^{\prime}(\Omega)\right]^{p}$ can be given. The first of these is that $\operatorname{det}(F)$ satisfy the Lojasiewicz inequality and that it not be identically zero in any component of $\Omega$. In fact, we have already proved this since the proof that (a) implies (d) in Theorem 3.1 makes no use of the hypothesis that $\Omega \subset R^{1}$. Thus we have

Proposition 4.1. Suppose $F=\left(f_{i j}\right)_{1 \leqq i, j \leqq p}$ where $f_{i j} \in \mathscr{E}(\Omega), \Omega \subset R^{n}$. If $\operatorname{im}(F)$ $=\left[\mathscr{D}^{\prime}(\Omega)\right]^{p}$, then $\operatorname{det}(F)$ is not identically zero in any component of $\Omega$ and for each compact set $K \subset \Omega$ there exists a constant $C>0$ and an integer $l \geqq 0$ such that

$$
|\operatorname{det}(F(x))| \geqq C[d(x, Z)]^{l} \text { for all } x \in K
$$

where $Z=\{x \in \Omega: \operatorname{det}(F(x))=0\}$. (Here $d(x, Z)=1$ for all $x$ if $Z=\varnothing$.)

Suppose $f \in \mathscr{E}(\Omega), \Omega \subset R^{n}$. A zero of $f$ is of finite order if some partial derivative of $f$ does not vanish at the zero. A second necessary condition for im $(F)=\left[\mathscr{D}^{\prime}(\Omega)\right]^{p}$ is that $\operatorname{det}(F)$ have zeros of finite order.

Proposition 4.2. Suppose $F=\left(f_{i j}\right)_{1 \leqq i, j \leqq p}$ where $f_{i j} \in \mathscr{E}(\Omega), \Omega \subset R^{n}$. If $\operatorname{im}(F)$ $=\left[\mathscr{D}^{\prime}(\Omega)\right]^{p}$, then the zeros of $\operatorname{det}(F)$ are of finite order.

Proof. Suppose $\operatorname{im}(F)=\left[\mathscr{D}^{\prime}(\Omega)\right]^{p}$. Then $\operatorname{im}\left(F_{c}\right)=\left[\mathscr{E}^{\prime}(\Omega)\right]^{p}$. We suppose that $\operatorname{det}(F)$ has a zero of infinite order, say at $a=\left(a_{1}, \ldots, a_{n}\right) \in \Omega$, and obtain $g_{1}, \ldots, g_{p} \in \mathscr{E}(\Omega)$ having zeros of infinite order at $a$ and $S_{1}, \ldots, S_{p}, T \in \mathscr{E}^{\prime}(\Omega)$ where $\operatorname{supp}(T)=\{a\}$ such that $g_{1} S_{1}+\cdots+g_{p} S_{p}=T$. This together with Malgrange's extension of Whitney's theorem leads to a contradiction.

If $f_{i j}$ has a zero of infinite order at $a$ for $1 \leqq i, j \leqq p$, then the fact that im $\left(F_{c}\right)$ $=\left[\mathscr{E}^{\prime}(\Omega)\right]^{p}$ implies that there exist $S_{1}, \ldots, S_{p} \in \mathscr{E}^{\prime}(\Omega)$ with

$$
f_{11} S_{1}+\cdots+f_{1 p} S_{p}=\delta_{a} .
$$


Otherwise, let $r$ be the largest integer such that there is an $r \times r$ submatrix of $F$ whose determinant is either nonzero at $a$ or has a zero of finite order at $a$. Then $1 \leqq r<p$. For simplicity assume that $\partial^{\alpha} \operatorname{det}\left(f_{i j}\right)_{1 \leqq i, j \leqq r}=\partial_{1}^{\alpha_{1}} \cdots \partial_{n}^{\alpha_{n}} \operatorname{det}\left(f_{i j}\right)_{1 \leqq i, j \leqq r}$ is nonzero at $a$. Then, proceeding as in the proof that (b) implies (c) in Theorem 2.1, we find $g_{1}, \ldots, g_{p} \in \mathscr{E}(\Omega)$ having zeros of infinite order at $a$ and $S_{1}, \ldots, S_{p} \in \mathscr{E}^{\prime}(\Omega)$ with

$$
g_{1} S_{1}+\cdots+g_{p} S_{p}=(-1)^{r} \operatorname{det}\left(f_{i j}\right)_{1 \leqq i, j \leqq r} \partial^{\alpha} \delta_{a} .
$$

Therefore there exist $g_{1}, \ldots, g_{p} \in \mathscr{E}(\Omega)$ having zeros of infinite order at $a$ and $S_{1}, \ldots, S_{p}, T \in \mathscr{E}^{\prime}(\Omega)$ where $\operatorname{supp}(T)=\{a\}$ such that $g_{1} S_{1}+\cdots+g_{p} S_{p}=T$.

Define $G_{c}:\left[\mathscr{E}^{\prime}(\Omega)\right]^{p} \rightarrow \mathscr{E}^{\prime}(\Omega)$ by $G_{c}\left(T_{1}, \ldots, T_{p}\right)=g_{1} T_{1}+\cdots+g_{p} T_{p}$, and let $G_{c}^{\prime}: \mathscr{E}(\Omega) \rightarrow[\mathscr{E}(\Omega)]^{p}$ be the transpose of $G_{c}$. Define $f$ by

$$
f\left(x_{1}, \ldots, x_{n}\right)=\left(x_{1}-a_{1}\right)^{l}+\cdots+\left(x_{n}-a_{n}\right)^{l}
$$

where $l$ is an even integer sufficiently large so that $f T=0$. Then $G_{c}\left(f S_{1}, \ldots, f S_{p}\right)$ $=f T=0$. Thus $\left(f S_{1}, \ldots, f S_{p}\right) \in \operatorname{ker}\left(G_{c}\right)=M^{\perp}$ where

$$
M=\operatorname{im}\left(G_{c}^{\prime}\right)=\left\{\left(g_{1} g, \ldots, g_{p} g\right): g \in \mathscr{E}(\Omega)\right\}
$$

is a submodule of the $\mathscr{E}(\Omega)$-module $[\mathscr{E}(\Omega)]^{p}$. Hence $\left(S_{1}, \ldots, S_{p}\right) \in(f M)^{\perp}$.

For $x \in \Omega$, let $J_{x}$ denote the ideal in $\mathscr{E}(\Omega)$ consisting of all functions which vanish at $x$ together with all derivatives and let $T_{x}$ denote the natural mapping of $[\mathscr{E}(\Omega)]^{p}$ onto $[\mathscr{E}(\Omega)]^{p} /\left[J_{x}\right]^{p}$. Then $T_{x}(f M)=T_{x}(M)$ for all $x \in \Omega$. By Whitney's theorem for submodules [4, Corollary 1.7 , p. 25] we conclude that $\mathrm{cl}(f M)=\mathrm{cl}(M)$ and hence that $(f M)^{\perp}=M^{\perp}$.

Therefore $\left(S_{1}, \ldots, S_{p}\right) \in M^{\perp}=\operatorname{ker}\left(G_{c}\right)$. But

$$
G_{c}\left(S_{1}, \ldots, S_{p}\right)=g_{1} S_{1}+\cdots+g_{p} S_{p}=T \neq 0 .
$$

This contradiction completes the proof of Proposition 4.2.

An example due to Malgrange [4, p. 89] shows that the converse of Proposition 4.2 is false for $\Omega \subset R^{n}, n>1$.

EXAMPLE 4.1. Let

$$
\begin{aligned}
f(x, y) & =e^{-1 / x^{2}}+y^{2}, & & x \neq 0, \\
& =y^{2}, & & x=0 .
\end{aligned}
$$

Then the zero of $f$ is of finite order. However, by Proposition 4.1, we see that $f \mathscr{D}^{\prime}\left(R^{2}\right) \neq \mathscr{D}^{\prime}\left(R^{2}\right)$ because $f$ fails to satisfy the Lojasiewicz inequality in any compact neighborhood of the origin.

The referee has pointed out an example which shows that the converse of Proposition 4.1 is false for $\Omega \subset R^{n}, n>1$.

EXAMPLE 4.2. Let $g(x, y)=y f(x, y)$ where $f$ is defined as in Example 4.1. Then $g$ satisfies the Lojasiewicz inequality, but

$$
g \mathscr{D}^{\prime}\left(R^{2}\right)=f y \mathscr{D}^{\prime}\left(R^{2}\right)=f \mathscr{D}^{\prime}\left(R^{2}\right) \neq \mathscr{D}^{\prime}\left(R^{2}\right) .
$$


5. General systems of division problems. For $F=\left(f_{i j}\right)_{1 \leqq i \leqq p, 1 \leqq j \leqq q}$ where $f_{i j} \in \mathscr{E}^{m}(\Omega), 0 \leqq m \leqq \infty, \Omega \subset R^{n}$, when does $\operatorname{im}(F)=\left[\mathscr{D}^{\prime m}(\Omega)\right]^{p}$ ? We first show that, for $p>q$, it is never the case that $\operatorname{im}(F)=\left[\mathscr{D}^{\prime m}(\Omega)\right]^{p}$. However, for $p \leqq q$, results analogous to Theorem 2.1, Theorem 3.1, and Proposition 4.2 hold.

Proposition 5.1. Suppose $F=\left(f_{i j}\right)_{1 \leqq i \leqq p, 1 \leqq j \leqq q}$ where $p>q$ and $f_{i j} \in \mathscr{E}^{m}(\Omega)$, $0 \leqq m \leqq \infty, \Omega \subset R^{n}$. Then $\operatorname{im}(F) \neq\left[\mathscr{D}^{\prime m}(\Omega)\right]^{p}$.

Proof. We prove that im $(F) \neq\left[\mathscr{D}^{\prime m}(\Omega)\right]^{p}$ by showing that

$$
F^{\prime}:\left[\mathscr{D}^{m}(\Omega)\right]^{p} \rightarrow\left[\mathscr{D}^{m}(\Omega)\right]^{q}
$$

is not one-to-one. Let $G$ be $F$ augmented by $p-q$ columns of zero functions, that is, let $G=\left(g_{i j}\right)_{1 \leqq i, j \leqq p}$ where $g_{i j}=f_{i j}$ for $1 \leqq i \leqq p, 1 \leqq j \leqq q$, and $g_{i j}=0$ for $1 \leqq i \leqq p$, $q<j \leqq p$. Then $\operatorname{det}(G)=0$ and therefore, by Lemma 3.1 ,

$$
G_{c}^{\prime}:\left[\mathscr{E}^{m}(\Omega)\right]^{p} \rightarrow\left[\mathscr{E}^{m}(\Omega)\right]^{p}
$$

is not one-to-one. Since $\operatorname{ker}\left(G_{c}^{\prime}\right)=\operatorname{ker}\left(F_{c}^{\prime}\right)$, we conclude that

$$
F_{c}^{\prime}:\left[\mathscr{E}^{m}(\Omega)\right]^{p} \rightarrow\left[\mathscr{E}^{m}(\Omega)\right]^{q}
$$

is not one-to-one. Hence $F^{\prime}$ is not one-to-one.

For $p \leqq q$, the analogue of Theorem 2.1 can be established by an argument similar to that employed in the proof of Theorem 2.1.

THEOREM 5.1. Suppose $F=\left(f_{i j}\right)_{1 \leqq i \leqq p, 1 \leqq j \leqq q}$ where $p \leqq q$ and $f_{i j} \in \mathscr{E}^{m}(\Omega), m<\infty$, $\Omega \subset R^{n}$. Then the following are equivalent:

(a) $\operatorname{im}(F)=\left[\mathscr{D}^{\prime m}(\Omega)\right]^{p}$.

(b) $\operatorname{im}\left(F_{c}\right)=\left[\mathscr{E}^{\prime m}(\Omega)\right]^{p}$.

(c) For every $x \in \Omega$ the rank of the matrix $F(x)$ is $p$.

Proof. (a) implies (b) as in Theorem 2.1.

Suppose $\operatorname{im}\left(F_{c}\right)=\left[\mathscr{E}^{\prime m}(\Omega)\right]^{p}$. By supposing that at some point $a \in \Omega$ the rank $r$ of the $p \times q$ matrix $F(a)$ is less than $p$ and arguing as in the proof that (b) implies (c) in Theorem 2.1, Lemma 2.1 is contradicted. Hence (b) implies (c).

Suppose that for every $x \in \Omega$ the rank of $F(x)$ is $p$. Then there is a locally finite open covering $\left\{\Omega_{\alpha}\right\}$ of $\Omega$ with the property that in each $\Omega_{\alpha}$ the determinant of some $p \times p$ submatrix of $F$ is never zero. Therefore, for $\left(T_{1}, \ldots, T_{p}\right) \in\left[\mathscr{D}^{\prime m}(\Omega)\right]^{p}$ with $\operatorname{supp}\left(T_{i}\right) \subset \Omega_{\alpha}$ for $1 \leqq i \leqq p$, there exists $\left(S_{1}, \ldots, S_{q}\right) \in\left[\mathscr{D}^{\prime m}(\Omega)\right]^{q}$ with $\operatorname{supp}\left(S_{j}\right) \subset \Omega_{\alpha}$ for $1 \leqq j \leqq q$ such that $F\left(S_{1}, \ldots, S_{q}\right)=\left(T_{1}, \ldots, T_{p}\right)$. Then, by using a partition of unity subordinate to the covering $\left\{\Omega_{\alpha}\right\}$, im $(F)=\left[\mathscr{D}^{\prime m}(\Omega)\right]^{p}$ is easily verified (see [7, p. 126]). Thus (c) implies (a).

The analogue of Theorem 3.1 is also valid for $p \leqq q$ but a method of proof different from that utilized in Theorem 3.1 seems to be required. 


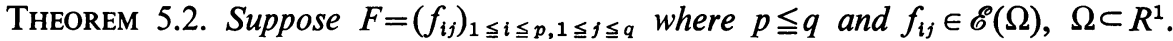
Let $\mathscr{F}$ be the set of $p \times p$ submatrices of $F$ and $Z=\{x \in \Omega$ : rank of $F(x)<p\}$ $=\{x \in \Omega: \operatorname{det}(M(x))=0$ for all $M \in \mathscr{F}\}$. Then the following are equivalent:

(a) $\operatorname{im}(F)=\left[\mathscr{D}^{\prime}(\Omega)\right]^{p}$.

(b) $\operatorname{im}\left(F_{c}\right)=\left[\mathscr{E}^{\prime}(\Omega)\right]^{p}$.

(c) For every $x \in Z$ there exists $M \in \mathscr{F}$ such that $x$ is a zero of finite order of $\operatorname{det}(M)$.

(d) $\max \{|\operatorname{det}(M)|: M \in \mathscr{F}\}$ is not identically zero in any component of $\Omega$ and satisfies the Lojasiewicz inequality, that is, for each compact set $K \subset \Omega$ there exists a constant $C>0$ and an integer $l \geqq 0$ such that

$$
\max \{|\operatorname{det}(M(x))|: M \in \mathscr{F}\} \geqq C[d(x, Z)]^{l} \text { for all } x \in K .
$$

(Here $d(x, Z)=1$ for all $x$ if $Z=\varnothing$.

Proof. (a) implies (b) as in Theorem 3.1.

Suppose $\operatorname{im}\left(F_{c}\right)=\left[\mathscr{E}^{\prime}(\Omega)\right]^{p}$. Suppose that there exists a point $a \in Z$ such that $\operatorname{det}(M)$ has a zero of infinite order at $a$ for every $M \in \mathscr{F}$. Proceeding as in the proof of Proposition 4.2, we obtain $g_{1}, \ldots, g_{q} \in \mathscr{E}(\Omega)$ having zeros of infinite order at $a$ and $S_{1}, \ldots, S_{q}, T \in \mathscr{E}^{\prime}(\Omega)$ where $\operatorname{supp}(T)=\{a\}$ such that $g_{1} S_{1}+\cdots+g_{q} S_{q}=T$. This together with Malgrange's extension of Whitney's theorem leads to a contradiction, as shown in the proof of Proposition 4.2. Hence (b) implies (c).

The equivalence of (c) and (d) follows from results of the author [6, Corollary 6.2].

To complete the proof, we show that (c) implies (a). Suppose that for every $x \in Z$ there exists $M \in \mathscr{F}$ such that $\operatorname{det}(M)$ has a zero of finite order at $x$. Then there is a locally finite open covering $\left\{\Omega_{\alpha}\right\}$ of $\Omega$ with the property that for each $\Omega_{\alpha}$ there exists $M \in \mathscr{F}$ such that det $(M)$ either has no zeros in $\Omega_{\alpha}$ or has one zero of finite order in $\Omega_{\alpha}$. Therefore, for $\left(T_{1}, \ldots, T_{p}\right) \in\left[\mathscr{D}^{\prime}(\Omega)\right]^{p}$ with $\operatorname{supp}\left(T_{i}\right) \subset \Omega_{\alpha}$ for $1 \leqq i \leqq p$, there exists $\left(S_{1}, \ldots, S_{q}\right) \in\left[\mathscr{D}^{\prime}(\Omega)\right]^{q}$ with $\operatorname{supp}\left(S_{j}\right) \subset \Omega_{\alpha}$ for $1 \leqq j \leqq q$ such that $F\left(S_{1}, \ldots, S_{q}\right)$ $=\left(T_{1}, \ldots, T_{p}\right)$. Here we are using the fact that division by a function with zeros of finite order is always possible in $R^{1}$ (see [7, p. 125] or [6, Corollary 6.2]). Then, using a partition of unity subordinate to the covering $\left\{\Omega_{\alpha}\right\}, \operatorname{im}(F)=\left[\mathscr{D}^{\prime}(\Omega)\right]^{p}$ is easily verified (see [7, p. 126]). Thus (c) implies (a).

For the general system of division problems in $\mathscr{D}^{\prime}(\Omega), \Omega \subset R^{n}$, the results of Malgrange [5, Theorem 1, p. 23-01] together with an extension of Lemma 3.1 imply that for $F=\left(f_{i j}\right)_{1 \leqq i \leqq p, 1 \leqq j \leqq q}$ where $p \leqq q$ and the $f_{i j}$ are real analytic in $\Omega$, if for each component of $\Omega$ there is a $p \times p$ submatrix of $F$ which is not identically zero in the component, then $\operatorname{im}(F)=\left[\mathscr{D}^{\prime}(\Omega)\right]^{p}$.

Observing that the proof that (a) implies (c) in Theorem 5.2 makes no use of the hypothesis that $\Omega \subset R^{1}$, we obtain the analogue of Proposition 4.2 for $p \leqq q$.

Proposition 5.2. Suppose $F=\left(f_{i j}\right)_{1 \leqq i \leqq p, 1 \leqq j \leqq q}$ where $p \leqq q$ and $f_{i j} \in \mathscr{E}(\Omega), \Omega \subset R^{n}$. If $\operatorname{im}(F)=\left[\mathscr{D}^{\prime}(\Omega)\right]^{p}$, then for every $x \in \Omega$ there exists a $p \times p$ submatrix of $F$ whose determinant is either nonzero at $x$ or has a zero of finite order at $x$. 
However, our proof of Theorem 5.2 does not establish the analogue of Proposition 4.1 for the general system of division problems.

\section{BIBLIOGRAPHY}

1. J. Dieudonné and L. Schwartz, La dualité dans les espaces $(\mathscr{F})$ et $(\mathscr{L} \mathscr{F})$, Ann. Inst. Fourier (Grenoble) 1 (1949), 61-101. MR 12, 417.

2. L. Hörmander, On the division of distributions by polynomials, Ark. Mat. 3 (1958), 555-568. MR 23 \#A2044.

3. S. Łojasiewicz, Sur le problème de la division, Studia Math. 18 (1959), 87-136. MR 21 \#5893.

4. B. Malgrange, Ideals of differentiable functions, Tata Institue of Fundamental Research Studies in Mathematics, no. 3, Tata Institute of Fundamental Research, Bombay; Oxford Univ. Press, London, 1967. MR 35 \#3446.

5. —_ Division des distributions, Séminaire Schwartz 1959/60, Faculté des Sciences, Paris, 1960, pp. 21-25. MR 23 \#A2275.

6. B. Roth, Finitely generated ideals of differentiable functions, Trans. Amer. Math. Soc. 150 (1970), 213-226.

7. L. Schwartz, Théorie des distributions, Hermann, Paris, 1966. MR 35 \#730.

8. H. Whitney, On ideals of differentiable functions, Amer. J. Math. 70 (1948), 635-658. MR 10, 126.

UNIVERSITY OF WYOMING,

LARAMIE, WYOMING 82070 